\title{
The cluster-galaxy cross spectrum
}

\section{An additional probe of cosmological and halo parameters}

\author{
G. Hütsi ${ }^{1,2}$ and O. Lahav ${ }^{1}$ \\ 1 Department of Physics and Astronomy, University College London, London WC1E 6BT, UK \\ e-mail: [ghutsi;lahav]@star.ucl.ac.uk \\ 2 Tartu Observatory, Tõravere 61602, Estonia
}

Received 23 May 2008 / Accepted 16 September 2008

\begin{abstract}
Context. There are several wide field galaxy and cluster surveys planned for the near future, e.g. BOSS, WFMOS, ADEPT, Hetdex, SPT, eROSITA. In the simplest approach, one would analyze these independently, thus neglecting the extra information provided by the cluster-galaxy cross pairs.

Aims. In this paper we have focused on the possible synergy between these surveys by investigating the amount of information encoded in the cross pairs.

Methods. We present a model for the cluster-galaxy cross spectrum within the halo model framework. To assess the gain in performance due to inclusion of the cluster-galaxy cross pairs, we carry out a Fisher matrix analysis for a BOSS-like galaxy redshift survey targeting luminous red galaxies and a hypothetical mass-limited cluster redshift survey with a lower mass threshold of $1.7 \times$ $10^{14} h^{-1} M_{\odot}$ over the same volume.

Results. On small scales, a cluster-galaxy cross spectrum directly probes the density profile of the halos, instead of the density profile convolved with itself, as is the case for the galaxy power spectrum. Due to this different behavior, adding information from the cross pairs helps to tighten constraints on the halo occupation distribution (e.g. a factor of $\sim 2$ compression of the error ellipses on the $m_{q}^{\text {low }}-\alpha$ plane) and offers an alternative mechanism compared with techniques that directly fit halo density profiles. By inclusion of the cross pairs, a factor of $\sim 2$ stronger constraints are obtained for $\sigma_{8}$, while the improvement for the dark energy figure-of-merit is somewhat weaker: an increase by a factor of 1.4 .

We have also written down the formalism for the case when only photometric redshifts are available for both the clusters and the galaxies. For the analysis of the photometric surveys the inclusion of the cluster-galaxy cross pairs might be very beneficial since the photo- $z$ errors for the clusters are usually significantly smaller than for the typical galaxies.
\end{abstract}

Key words. cosmology: theory - large-scale structure of Universe - galaxies: clusters: general - galaxies: statistics cosmological parameters

\section{Introduction}

With the advent of the modern wide-field spectroscopic sky surveys, which have measured redshifts for hundreds of thousands of galaxies, e.g. $2 \mathrm{dFGRS}{ }^{1}, \mathrm{SDSS}^{2}$, we have gained a good picture of the cosmic large-scale structure out to redshifts of $\sim 0.5$. From the measurements of the cosmic microwave background angular temperature fluctuations (e.g. Hinshaw et al. 2008), we have obtained a good understanding of the nature and statistics of the small density fluctuations that have evolved through gravitational instability to the filament-void network of the matter surrounding us at low redshifts. These measurements have proven that initial fluctuations are compatible of being adiabatic and Gaussian with roughly the scale-free initial spectrum. Because the initial fluctuations can be described as a Gaussian random field, the full information is contained in the two-point correlator of the field. Under the linear evolution of the initial fluctuation field, which is valid on large scales $\left(k \lesssim 0.1 h \mathrm{Mpc}^{-1}\right)$, the

\footnotetext{
${ }^{1}$ http://wWw.mso.anu.edu.au/2dFGRS/

${ }^{2}$ http://www.sdss.org/
}

Gaussian field remains Gaussian in nature, and as such explains the central interest in measuring the clustering power spectrum (or its real space analog - two-point correlation function) using the high-quality data from the above-mentioned spectroscopic surveys. Data from the 2dFGRS and from the SDSS survey have both led to the high-precision measurement of the low-redshift galaxy power spectra (Percival et al. 2001; Tegmark et al. 2004; Cole et al. 2005; Tegmark et al. 2006). Moreover, these datasets have proven to be powerful enough to enable one to detect theoretically expected small fluctuations ( $\sim 5 \%$ relative amplitude $)-$ baryonic acoustic oscillations (BAO) - in the clustering power spectra (Eisenstein et al. 2005; Cole et al. 2005; Hütsi 2006a; Padmanabhan et al. 2007; Blake et al. 2007; Tegmark et al. 2006; Percival et al. 2007). The realization that BAO can be used as a standard ruler to map the low-redshift expansion history of the Universe (Eisenstein \& Hu 1998; Blake \& Glazebrook 2003; Hu \& Haiman 2003; Linder 2003; Seo \& Eisenstein 2003), and thus to shed light on the nature of the mysterious dark energy (DE), has led several groups to propose next generation redshift surveys that should cover even larger sky areas and go significantly deeper in redshift. A few examples of the 
proposed projects include $\mathrm{BOSS}^{3}, \mathrm{WFMOS}^{4}, \mathrm{ADEPT}^{5}$, Hetdex $^{6}$, and also already ongoing $\mathrm{WiggleZ}^{7}$ project performed on Anglo-Australian telescope.

In addition to these galaxy redshift surveys, a fast development in the microwave detector technology has led to the possibility of detecting tens of thousands of galaxy clusters over several thousand square degrees through thermal SunyaevZeldovich (SZ) effect (Sunyaev \& Zeldovich 1972, 1980). Currently the most ambitious among these projects is the ongoing $\mathrm{SPT}^{8}$ survey. Also there are plans in the X-ray community to carry out a next-generation X-ray cluster survey with the yield of $\sim 100000$ galaxy clusters (eROSITA ${ }^{9}$ survey). With a further possibility of obtaining spectroscopic redshifts for the SZ/X-ray selected galaxy clusters through the optical follow-up, one can turn these large cluster samples into an extremely powerful cosmological probe.

In this paper we study the possible synergy between these galaxy and galaxy cluster surveys, focusing particularly on the two-point clustering measures. As the simplest approach, one would analyze these surveys independently, i.e. concentrating separately on galaxy-galaxy and cluster-cluster pairs. However, this approach would neglect extra information provided by the cluster-galaxy cross pairs. In fact, the study of the cluster-galaxy cross correlations has a fairly long history, (e.g. Peebles 1974; Seldner \& Peebles 1977a,b; Lilje \& Efstathiou 1988). Although rather sparse, cluster samples with strongly increased clustering strength as compared to the galaxies, had proven to be powerful tracers of the large-scale structure of the Universe. Initially the main interest in determining the cluster-galaxy cross correlation function was driven by the desire to reduce the shot noise level inherent in these relatively sparse cluster samples.

As the main task of this work we are going to determine the information gain provided by the cluster-galaxy cross spectrum $\left(P_{c-g}\right)$ over the information encoded in the galaxy-galaxy and cluster-cluster spectra $\left(P_{g-g}\right.$ and $P_{c-c}$, respectively). We carried out our modeling using a halo model (HM) (e.g. Seljak 2000, 2001; Cooray \& Sheth 2002) approach. To our knowledge this is the first time that HM has been used to model $P_{c-g}$. As such, we try to give a comprehensive explanation of the results obtained. We applied the derived formalism to a BOSSlike galaxy survey covering $10000 \mathrm{deg}^{2}$ and reaching redshifts of 0.7 . For the cluster survey we assumed that it covers the same volume as the galaxy survey and is simply mass-limited with the lower mass cut-off $m_{c}^{\text {low }}=1.7 \times 10^{14} h^{-1} M_{\odot}$, which is close to the mass limit obtainable by the SPT. In reality BOSS and SPT surveys do not overlap, but eROSITA, which has roughly the same effective sensitivity (although the threshold mass in this case depends more strongly on redshift) is a full-sky cluster survey, and thus completely overlaps with BOSS.

In our study we focus on two-point clustering statistics. Thus our approach is complementary to the number count analysis. In the case of the galaxy cluster samples, the complementarity of the number count and clustering analysis has been investigated by Majumdar \& Mohr (2004).

\footnotetext{
3 http://cosmology.1bl.gov/BOSS/

${ }^{4}$ http://arxiv.org/ftp/astro-ph/papers/0510/0510272. pdf

5 E.g.http://www.science.doe.gov/hep/hepap/feb2007/ hepap_bennett_febo7.pdf

${ }^{6}$ http://www.as. utexas. edu/hetdex/

7 http://wigglez.swin.edu.au/

8 http://pole.uchicago.edu/

9 http://www.mpe.mpg.de/erosita/MDD-6.pdf
}

Our paper is organized as follows. In Sect. 2 we present the formalism, which is applied to hypothetical galaxy and cluster cluster surveys in Sect. 3. There the main focus is to find out the gain in information once cluster-galaxy cross pairs are included. Finally, Sect. 4 brings our conclusions.

Throughout the paper we assume a fiducial cosmology to be a flat "concordance" $\Lambda$ CDM model (Bahcall et al. 1999) with $\Omega_{\mathrm{m}}=0.27, \Omega_{\mathrm{b}}=0.045, h=0.7$, and $\sigma_{8}=0.85$. All these parameter values, except for the somewhat higher value for $\sigma_{8}$, are in good agreement with the recent WMAP5 results (Dunkley et al. 2008).

\section{Halo model power spectra. Covariances and Fisher matrices}

\subsection{Power spectra in real space}

In this section we present the results for the matter $(m-m)$, galaxy $(g-g)$, cluster $(c-c)$, and cluster-galaxy $(c-g)$ power spectra in real space within the framework of the HM. The corresponding results for the redshift space are given in the following section. For a comprehensive review of HM see e.g. Cooray \& Sheth (2002). To those readers familiar with the HM, we suggest jumping directly to Eqs. (5)-(8), which present a commonly used formulation of the $g-g$ power spectrum in real space. Our formalism for the $c-c$ and $c-g$ spectra in real space is new and given by Eqs. (13)-(18). Equations (23)-(34) provide analogous formulae for the anisotropic spectra in redshift space.

In $\mathrm{HM}$, the power spectra can be expressed as a sum of the one-halo $(1 h)$ and two-halo $(2 h)$ terms:

$P_{x}=P_{x}^{1 h}+P_{x}^{2 h}$

which provide the contributions of pairs of points inhabiting the same or separate dark matter halos, respectively. For our cases of interest the subscript $x$ can be either $m-m, g-g, c-c$, or $c-g$.

Following Seljak (2000) and Cooray \& Sheth (2002), the $1 h$ and $2 h$ terms for the matter power spectrum at redshift $z$ can be expressed as

$$
\begin{aligned}
& P_{m-m}^{1 h}(k \mid z)=\int \mathrm{d} m n(m \mid z)\left(\frac{m}{\bar{\rho}}\right)^{2} u_{m}^{2}(k \mid m, z), \\
& P_{m-m}^{2 h}(k \mid z)=b_{m}^{2}(k \mid z) g^{2}(z) P^{\operatorname{lin}}(k \mid z=0), \\
& b_{m}(k \mid z) \equiv \int \mathrm{d} m n(m \mid z) b(m \mid z)\left(\frac{m}{\bar{\rho}}\right) u_{m}(k \mid m, z) .
\end{aligned}
$$

In the above expressions, $n(m \mid z)$ is the mass function and $b(m \mid z)$ halo bias parameter at redshift $z$. We calculated these using prescriptions by Sheth \& Tormen (1999) and Sheth et al. (2001). The function $g(z)$ represents linear growth factor at redshift $z$ normalized such that $g(z=0)=1$, and $\bar{\rho} \equiv \Omega_{\mathrm{m}} \rho_{\mathrm{c}}=$ $\int \mathrm{d} m m n(m \mid z) b(m \mid z)$ gives the mean comoving matter density. The quantity $u_{m}(k \mid m, z)$ is the normalized (i.e. $\lim _{k \rightarrow 0} u_{m}(k \mid m, z)=1$ ) Fourier transform of the dark matter density profile within a halo of mass $m$ at redshift $z$, which we take to be given by the the NFW (Navarro et al. 1997) form. The concentration parameter of the density profiles and its evolution with redshift is assumed to take an analytic form as given in Bullock et al. (2001). To calculate the linear power spectrum $P^{\operatorname{lin}}(k \mid z=0)$, we assume an initial power-law spectrum with spectral index $n_{\mathrm{s}}=1$ and use fitting formulae for transfer functions $T(k)$ as given by Eisenstein \& $\mathrm{Hu}$ (1998). 
The corresponding results for the $g-g$ case can be expressed as (see Cooray \& Sheth 2002, for details)

$$
\begin{aligned}
& P_{g-g}^{1 h}(k \mid z)=\int \mathrm{d} m n(m \mid z) \frac{\left\langle N_{g}\left(N_{g}-1\right) \mid m\right\rangle}{\bar{n}_{g}^{2}(z)}\left|u_{g}(k \mid m, z)\right|^{p}, \\
& P_{g-g}^{2 h}(k \mid z)=b_{g}^{2}(k \mid z) g^{2}(z) P^{\mathrm{lin}}(k \mid z=0), \\
& b_{g}(k \mid z) \equiv \int \mathrm{d} m n(m \mid z) b(m \mid z) \frac{\left\langle N_{g} \mid m\right\rangle}{\bar{n}_{g}(z)} u_{g}(k \mid m, z), \\
& \bar{n}_{g}(z) \equiv \int \mathrm{d} m n(m \mid z)\left\langle N_{g} \mid m\right\rangle .
\end{aligned}
$$

These results follow directly from the corresponding results for the $m-m$ case if one imagines the mass density field as composed of discrete particles, and thus can write $m / \bar{\rho} \equiv N / \bar{n}$ where $N$ is the number of particles in a halo with mass $m$, and $\bar{n}$ is the average number density of particles. To count the number of pairs correctly in this discrete case, we have a second factorial moment (instead of simply a second moment) of the halo occupation distribution (HOD) in Eq. (5). In the above relations, $\bar{n}_{g}(z)$ is the mean number density of galaxies, and $u_{g}(k \mid m, z)$ is the normalized Fourier transform of the galaxy number density profile within a halo of mass $m$ at redshift $z$. For $u_{g}(k \mid m, z)$ we assume that it follows the corresponding density distribution for the dark matter; i.e., we take $u_{g}(k \mid m, z)=u_{m}(k \mid m, z)$. We further assume that the 1st and 2nd factorial moments of the HOD are given by the following parametric forms (e.g. Cooray 2004)

$$
\begin{aligned}
& \left\langle N_{g} \mid m\right\rangle=\left\{\begin{array}{ccc}
\left(\frac{m}{m_{0}}\right)^{\alpha} & \text { if } & m \geq m_{g}^{\text {low }} \\
0 & \text { if } & m<m_{g}^{\text {low }},
\end{array}\right. \\
& \left\langle N_{g}\left(N_{g}-1\right) \mid m\right\rangle=\beta^{2}(m)\left\langle N_{g} \mid m\right\rangle^{2}, \\
& \beta(m)= \begin{cases}\frac{1}{2} \log \left(\frac{m}{10^{11} h^{-1} M_{\odot}}\right) & \text { if } m<10^{13} h^{-1} M_{\odot} \\
1 & \text { otherwise. }\end{cases}
\end{aligned}
$$

This HOD model is consistent with assuming that for the highmass halos $\left(m>10^{13} h^{-1} M_{\odot}\right)$, the HOD follows Poisson distribution, whereas the fluctuations in $N_{g}$ are sub-Poissonian for the lower masses. Thus in our case we have three free parameters describing the HOD: $\alpha, m_{0}$, and $m_{g}^{\text {low }}$.

Here we have made an assumption that, if there are any galaxies in a halo, then one of these is located in the central position. Thus for the $1 h$ term, we have to deal with two types of pairs: (i) ones that involve the central object, and so probe the density profile of the halo directly (i.e. the power law index $p=1$ in Eq. (5)); (ii) all the other pairs, which describe the convolution of the density profile with itself (i.e. $p=2$ ). The detailed treatment (see e.g. Cooray \& Sheth 2002) of the central objects leads to the following choice for $p$ in Eq. (5)

$p= \begin{cases}1 & \text { if } \quad\left\langle N_{g}\left(N_{g}-1\right) \mid m\right\rangle<1 \\ 2 & \text { if } \quad\left\langle N_{g}\left(N_{g}-1\right) \mid m\right\rangle \geq 1 .\end{cases}$

Following the derivations for the matter and galaxy power spectra as given in Cooray \& Sheth (2002), we obtain the corresponding results for the $c-c$ and $c-g$ cases. The cluster power spectrum can be given as

$$
\begin{aligned}
& P_{c-c}^{1 h}(k \mid z)=0 \\
& P_{c-c}^{2 h}(k \mid z)=b_{c}^{2}(k \mid z) g^{2}(z) P^{\operatorname{lin}}(k \mid z=0), \\
& b_{c}(k \mid z) \equiv \frac{1}{\bar{n}_{c}(z)} \int_{m_{c}^{\text {low }}(z)} \mathrm{d} m n(m \mid z) b(m \mid z), \\
& \bar{n}_{c}(z) \equiv \int_{m_{c}^{\text {low }}(z)} \mathrm{d} m n(m \mid z)
\end{aligned}
$$

and the cluster-galaxy cross spectrum as

$$
\begin{aligned}
& P_{c-g}^{1 h}(k \mid z)=\frac{1}{\bar{n}_{c}(z) \bar{n}_{g}(z)} \int_{m_{c}^{\mathrm{low}}(z)} \mathrm{d} m n(m \mid z)\left\langle N_{g} \mid m\right\rangle u_{g}(k \mid m, z), \\
& P_{c-g}^{2 h}(k \mid z)=b_{c}(k \mid z) b_{g}(k \mid z) g^{2}(z) P^{\mathrm{lin}}(k \mid z=0) .
\end{aligned}
$$

It is clear that there is no $1 h$ term for the cluster power spectrum $^{10}$, whereas the $2 h$ term can be obtained from the corresponding $g-g 2 h$ term by replacing $\left\langle N_{g} \mid m\right\rangle$ with 1 (i.e. each dark matter halo hosts one cluster), $\bar{n}_{g}(z)$ with $\bar{n}_{c}(z)$, and taking the normalized Fourier transform of the density profile ( $\delta$-function in our case) equal to $1^{11}$. For calculating cluster power spectra, we assume for simplicity that our cluster sample is mass-selected above some mass limit $m_{c}^{\text {low }}$ that can depend on redshift.

On small scales (i.e. relevant for the $1 h$ term), cluster-galaxy cross correlations probe density profile around the halo center i.e. in comparison to the $g-g$ case, where power-law index $p$ in Eq. (5) can take the value 2, but here we have only a linear dependence on $u_{g}(k \mid m, z)$. The number of pairs contributing to the $1 h$ term is simply given by the number of galaxies within each halo i.e. $\left\langle N_{g} \mid m\right\rangle$.

In the above formulae we have used the notations $b_{m}(k \mid z)$, $b_{g}(k \mid z)$, and $b_{c}(k \mid z)$ to emphasize that these functions give the effective bias parameters for large scales (i.e. neglecting the contributions from $1 h$ terms). From Eq. (18) we see that the effective large-scale bias parameter $b_{c-g}(k \mid z)$ for the cross spectrum is given by $\sqrt{b_{c}(k \mid z) b_{g}(k \mid z)}$, as expected.

Examples of the real-space spectra and bias parameters are given in the left-hand panels of Fig. 1. The results presented there correspond to the redshift $z=0.5$. To calculate the cluster spectrum, we assumed a mass-limited sample of clusters above a threshold mass $m_{c}^{\text {low }}=1.7 \times 10^{14} h^{-1} M_{\odot}$. The parameters for the galaxy HOD were fixed as follows: $\alpha=0.9, m_{g}^{\text {low }}=5 \times$ $10^{12} h^{-1} M_{\odot}$ and $m_{0}=4.2 \times 10^{13} h^{-1} M_{\odot}$. For the motivation of these values see Sect. 3 . Where relevant we have also shown contributions from the $1 h$ and $2 h$ terms separately. Here it is worth noticing a relatively greater importance of the $1 h$ term in the case of the cross spectrum as compared to the galaxy-galaxy power spectrum. In the lower panel we have used a superscript "tot" to denote a total bias parameter, which includes both $1 h$ and $2 h$ contributions, in contrast to the bias parameters $b_{g}, b_{c}$ (see Eqs. (7), (15)), and $b_{c g}=\sqrt{b_{g} b_{c}}$, which only incorporate $2 h$ contributions. For low values of the wavenumber $k$, the total bias parameters deviate slightly from the results obtained by using $2 h$ terms only due to the large-scale "leakage" of the $1 h$ term $^{12}$.

\footnotetext{
${ }_{10}$ Here we chose to subtract the shot noise term, which effectively corresponds to the cluster number counts. Thus our clustering analysis is independent and complementary to the number-count studies.

11 To be more precise, even in Eq. (7) one has to take $u_{g}(k \mid m, z)=1$ if $\left\langle N_{g} \mid m\right\rangle \leq 1$, since as we assumed, if there is one galaxy per halo, it must be located at the central position. In reality, however, these details make a negligible difference.

12 It is clear that these additional Poissonian fluctuations on the largest scales are incompatible with the large-scale homogeneity requirement. This is a shortcoming of the HM that has not been solved satisfactorily so far. A possible way around it would be to use compensated density profiles for the halos, i.e. no DC component in their Fourier transforms. Even then, in $2 h$ terms one still has to use uncompensated profiles, since convolving with the profile with zero DC component would damp out the large-scale power completely. However, the deviations of the bias parameters are fairly small for the scales of interest in this paper, and thus we do not make any attempt to correct for this shortcoming.
} 

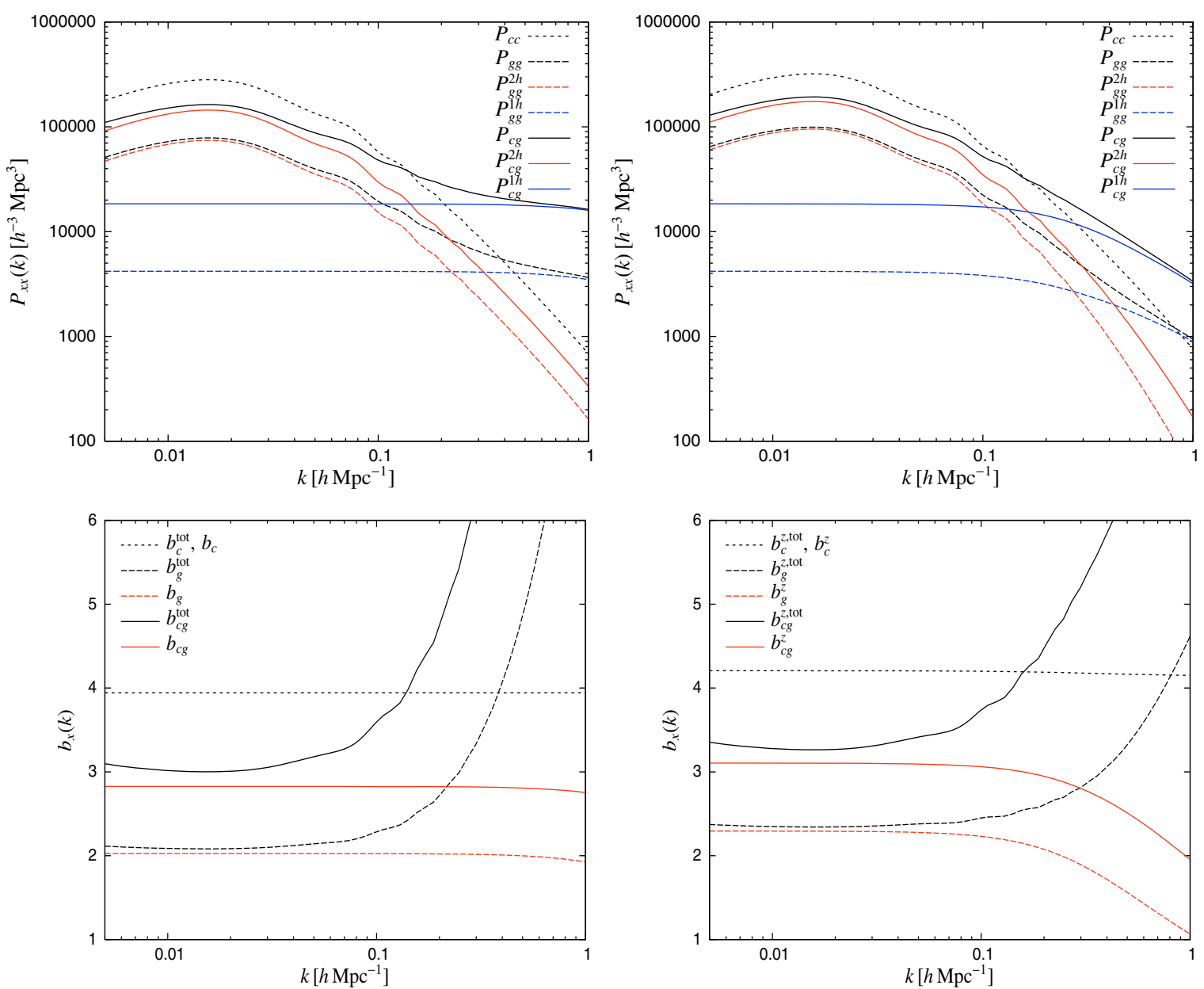

Fig. 1. Power spectra and bias parameters in real (left-hand panels) and redshift space (right-hand panels). The short-dashed, dashed, and solid lines correspond to cluster, galaxy, and cross spectrum, respectively. Where relevant we have shown contributions from the $1 h$ and $2 h$ terms separately. In lower panels a superscript "tot" is used to denote a total bias parameter, which includes both $1 h$ and $2 h$ contributions, in contrast to the bias parameters $b_{g}, b_{c}$, and $b_{c g}=\sqrt{b_{g} b_{c}}$, which incorporate $2 h$ contributions only. See the main text for the assumed values of the HOD and other parameters.

\section{2. redshift-space power spectra}

In this section we are going to generalize the previous results to include the redshift-space distortions. Since in addition to the above-mentioned future redshift surveys, there are several imaging surveys planned (e.g. DES ${ }^{13}$, Pan-STARRS ${ }^{14}$, DUNE $^{15}$, $\mathrm{LSST}^{16}$ ), in Appendix A we also give a simple treatment in case only photometric redshifts are available. Here we partially follow the formalism as given in White (2001) and in Seljak (2001). For the results presented in this section, we assume a flat-sky approximation.

Small-scale redshift-space distortions ("Fingers-of-God" (FOG)) in coordinate space can be modeled as a convolution along the line of sight with a 1D Gaussian kernel. As Gaussian also stays Gaussian in Fourier space, the matter density contrast gets modified as

$\delta_{\boldsymbol{k}}^{m} \rightarrow \delta_{\boldsymbol{k}}^{m} \cdot \mathcal{F}\left(k \sigma_{m} \mu\right)$,

\footnotetext{
13 https://www . darkenergysurvey.org/

14 http://pan-starrs.ifa.hawaii.edu/public/

15 http://www. dune-mission.net/

16 http://www. lsst.org/
}

where the damping factor $\mathcal{F}$ is given by

$$
\mathcal{F}(x) \equiv \exp \left(-\frac{x^{2}}{2}\right) \text {. }
$$

Here $\mu$ is the cosine of the angle between the line of sight direction $\hat{\boldsymbol{r}}$ and wavevector $\boldsymbol{k}$, i.e. $\mu \equiv \hat{\boldsymbol{r}} \cdot \hat{\boldsymbol{k}}$. As in Cooray (2004), we assume that the one-dimensional velocity dispersion of matter inside a halo with mass $m$ at redshift $z, \sigma_{m}(m, z)$, follows the scaling of the isothermal sphere model:

$\sigma_{m}(m, z)=\gamma \cdot \sqrt{\frac{G m}{2 r_{\mathrm{vir}}(m, z)}}$,

where $r_{\mathrm{vir}}$ is the virial radius of the halo and $\gamma$ is a free parameter, which in the following we keep fixed to 1, i.e. precisely assume the value for the isothermal sphere model. The corresponding velocity dispersion of galaxies is taken to follow the same relation.

There are additional redshift-space distortions on large scales due to the coherent inflows of matter towards massive accretion centers. This leads to modifying the density fluctuations in Fourier space, such that (Kaiser 1987)

$\delta_{\boldsymbol{k}}^{x} \rightarrow \delta_{\boldsymbol{k}}^{x}+f \cdot \delta_{\boldsymbol{k}}^{m} \mu^{2}$, 
where $f$ represents the logarithmic derivative of the growth factor $g(z)$, i.e. $f \equiv \mathrm{d} \log g / \mathrm{d} \log a$, and superscript $x$ can be either $m$ (for matter), $c$ (clusters), or $g$ (galaxies).

Taking into account both the small and large-scale distortions, we can write down the results for the anisotropic power spectra as follows

$P_{c-c}^{1 h}(k, \mu \mid z)=0$,

$P_{g-g}^{1 h}(k, \mu \mid z)=\int \mathrm{d} m n(m \mid z) \frac{\left\langle N_{g}\left(N_{g}-1\right) \mid m\right\rangle}{\bar{n}_{g}^{2}(z)}\left|u_{g}(k \mid m, z)\right|^{p}$ $\mathscr{F}^{p}\left[k \sigma_{m}(m, z) \mu\right]$,

$P_{c-g}^{1 h}(k, \mu \mid z)=\frac{1}{\bar{n}_{c}(z) \bar{n}_{g}(z)} \int_{m_{c}^{\mathrm{low}}(z)} \mathrm{d} m n(m \mid z)\left\langle N_{g} \mid m\right\rangle u_{g}(k \mid m, z)$ $\mathscr{F}\left[k \sigma_{m}(m, z) \mu\right]$,

$P_{c-c}^{2 h}(k, \mu \mid z)=b_{c}^{z}(k, \mu \mid z)^{2} g^{2}(z) P^{\operatorname{lin}}(k \mid z=0)$,

$P_{g-g}^{2 h}(k, \mu \mid z)=b_{g}^{z}(k, \mu \mid z)^{2} g^{2}(z) P^{\operatorname{lin}}(k \mid z=0)$,

$P_{c-g}^{2 h}(k, \mu \mid z)=b_{c g}^{z}(k, \mu \mid z)^{2} g^{2}(z) P^{\operatorname{lin}}(k \mid z=0)$,

$b_{c}^{z}(k, \mu \mid z) \equiv b_{c}(k, \mu \mid z)+f(z) b_{m}(k, \mu \mid z) \mu^{2}$

$b_{g}^{z}(k, \mu \mid z) \equiv b_{g}(k, \mu \mid z)+f(z) b_{m}(k, \mu \mid z) \mu^{2}$

$b_{c g}^{z}(k, \mu \mid z) \equiv \sqrt{b_{c}^{z}(k, \mu \mid z) b_{g}^{z}(k, \mu \mid z)}$

$b_{c}(k, \mu \mid z) \equiv \frac{1}{\bar{n}_{c}(z)} \int_{m_{c}^{\mathrm{low}}(z)} \mathrm{d} m n(m \mid z) b(m \mid z)$,

$b_{g}(k, \mu \mid z) \equiv \int \mathrm{d} m n(m \mid z) b(m \mid z) \frac{\left\langle N_{g} \mid m\right\rangle}{\bar{n}_{g}(z)} u_{g}(k \mid m, z)$

$$
\mathscr{F}\left[k \sigma_{m}(m, z) \mu\right],
$$

$b_{m}(k, \mu \mid z) \equiv \int \mathrm{d} m n(m \mid z) b(m \mid z)\left(\frac{m}{\bar{\rho}}\right) u_{m}(k \mid m, z)$

$$
\cdot \mathcal{F}\left[k \sigma_{m}(m, z) \mu\right] .
$$

Examples of the spectroscopic redshift-space spectra and bias parameters are given in the right-hand panels of Fig. 1. The HOD and other parameters are exactly the same as in the left-hand panels (see the end of Sect. 2.1 for details), which show the corresponding results for the real space. As before, a superscript "tot" in the lower panel denotes a total bias parameter, which includes both $1 h$ and $2 h$ contributions, in contrast to the bias parameters $b_{c}^{z}, b_{g}^{z}$, and $b_{c g}^{z}$ (see Eqs. (29)-(31)), which incorporate $2 h$ contributions only. The spectra and bias parameters presented here are the angle-averaged versions. Note the expected largescale boost and small-scale damping of power as compared to the equivalent real-space results.

\subsection{Cosmological distortion}

The cosmological distortion (Alcock \& Paczynski 1979) arises simply because conversion of the observed redshifts to comoving distances requires specifying the cosmological model. If this cosmology differs from the true one, we are left with additional distortion of distances along and perpendicular to the line of sight. In general, the spatial power-spectrum measurements, in contrast to the angular spectra, are model-dependent; i.e., along with the measurements of the 3D power spectrum, one always has to specify the so-called fiducial model used to analyze the data. In principle, for each of the assumed cosmological models, one should redo the full power spectrum analysis to accommodate different distance-redshift relations. However, there is an easier way around this by finding an approximate analytical transformation that describes how the model spectrum should look under the distance-redshift relation given by the fiducial model. This transformation for the power spectrum is different along and perpendicular to the line of sight, and is also dependent on redshift. For more discussion of these issues see e.g. Hütsi (2006b). The observed spectrum $\widetilde{P}_{x}$ under the assumed distance-redshift relation of the fiducial cosmological model is related to the true spectrum $P_{x}$ as (Hütsi 2006b)

$\widetilde{P}_{x}\left(k_{\|}, k_{\perp} \mid z\right)=\frac{1}{c_{\|}(z) \cdot c_{\perp}^{2}(z)} \cdot P_{x}\left[\frac{k_{\|}}{c_{\|}(z)}, \frac{k_{\perp}}{c_{\perp}(z)} \mid z\right]$,

where the distortion parameters along and perpendicular to the line of sight are given as

$c_{\|}(z)=\frac{H^{\mathrm{fid}}(z)}{H(z)}$,
$c_{\perp}(z)=\frac{d_{\perp}(z)}{d_{\perp}^{\mathrm{fid}}(z)}$.

Here $H(z)$ denotes the Hubble parameter, and $d_{\perp}(z)$ is the comoving angular diameter distance. Superscript fid refers to the fiducial model. Here and in the following, we use a tilde on top of $P_{x}$ to denote theoretical spectrum "transformed to the reference frame of the fiducial cosmology". As we use the spectra that have the dimensions of volume an extra division by $c_{\|}(z) \cdot c_{\perp}^{2}(z)$ occurs due to the transformation of the volume elements. The same transformation in terms of $k=\left(k_{\|}^{2}+k_{\perp}^{2}\right)^{1 / 2}$ and $\mu=k_{\|} / k$ can be expressed as

$\widetilde{P}_{x}(k, \mu \mid z)=\frac{1}{c_{\|}(z) \cdot c_{\perp}^{2}(z)} \cdot P_{x}\left[\frac{\alpha(\mu, z)}{c_{\perp}(z)} k, \frac{1}{\varkappa(z) \alpha(\mu, z)} \mu \mid z\right]$,

where

$\varkappa(z) \equiv \frac{c_{\|}(z)}{c_{\perp}(z)}$

$\alpha(\mu, z) \equiv \sqrt{1+\left(\frac{1}{x^{2}(z)}-1\right) \mu^{2}}$

\subsection{Covariances and Fisher matrices}

Having an HM description for the various spectra, we can go on to calculate covariances and Fisher matrices. We give all the necessary details in Appendix B.

\section{Application of the formalism}

In this section we are going to apply the machinery presented in the previous section to the hypothetical ideal galaxy and galaxy cluster redshift surveys. For the galaxy survey, we assume specifications similar to the planned BOSS survey: $10000 \mathrm{deg}^{2}$ sky coverage, redshift range $z=0.1-0.7$, and the comoving number density of galaxies $\sim 3 \times 10^{-4} h^{3} \mathrm{Mpc}^{-3}$. Thus the volume of the survey is $\sim 5.7 h^{-3} \mathrm{Gpc}^{3}$ with the total number of galaxies reaching $\sim 1700000$. As mentioned in Sect. 2, we assume a simple HOD given by Eq. (9); i.e., we have three free parameters: $\alpha, m_{0}$, and $m_{g}^{\text {low }}$. For the parameter $\alpha$ we choose a fiducial value of 0.9 , which is typical for the red galaxies according to the semi-analytic galaxy formation models (see e.g. 
Cooray \& Sheth 2002) ${ }^{17}$. Looking at the equations given in the previous section, it is evident that power spectra do not depend on $m_{0}$. Thus after fixing the value for $\alpha$ the fiducial value for the parameter, $m_{g}^{\text {low }}$ can be chosen so as to give a clustering strength typical of the luminous red galaxies targeted by BOSS, i.e. the product of the bias and linear growth factor $b(z) g(z) \sim 2$. This gives us $m_{g}^{\text {low }} \simeq 5 \times 10^{12} h^{-1} M_{\odot}$. Once $\alpha$ and $m_{g}^{\text {low }}$ are fixed, the fiducial value for $m_{0}$ is obtained through the number density constraint, giving $m_{0} \simeq 4.2 \times 10^{13} h^{-1} M_{\odot}$. The total survey volume is divided into six redshift bins with a width $\Delta z=0.1$.

The galaxy cluster survey is assumed to cover exactly the same volume. For simplicity we model our cluster survey as a mass-limited survey above a threshold mass of $1.7 \times$ $10^{14} h^{-1} M_{\odot}$. This is very similar to the mass sensitivity obtainable by the SPT survey. Of course, one could model the cluster selection (e.g. SZ or X-ray selection) more realistically by taking the threshold mass to be an appropriate function of redshift. However, for clarity we have chosen to keep things as simple as possible. As an additional prior, we assume that the threshold mass can be calibrated with an accuracy of $10 \%$. It turns out that the information in the cluster-galaxy cross spectrum itself helps in determining the threshold mass with an accuracy of $\sim 4 \%$, so our results are rather insensitive to this assumed prior.

In addition to the HM parameters, we have to fix a fiducial cosmological model. This we take to be a flat $\Lambda \mathrm{CDM}$ model with $\Omega_{\mathrm{m}}=0.27, \Omega_{\mathrm{b}}=0.045, h=0.7$, and $\sigma_{8}=$ 0.85 . We focus only on spatially flat models in our Fisher matrix calculations. For the dark-energy equation of state we use a common $w_{0}-w_{a}$ parametrization (Chevallier \& Polarski 2001): $w(a)=w_{0}+(1-a) w_{a}(a=1 /(1+z))$, so our parameter vector $\boldsymbol{\Theta}$ has nine components in total: $\boldsymbol{\Theta}=\left(\Omega_{m}, \Omega_{\mathrm{b}}, h, \sigma_{8}, w_{0}, w_{a}, \ln m_{g}^{\text {low }}, \alpha, \ln m_{c}^{\text {low }}\right)$ with the following fiducial values: $\boldsymbol{\Theta}^{\text {fid }}=(0.27,0.045,0.7,0.85,-1 ., 0 ., \ln (5 \times$ $\left.\left.10^{12}\right), 0.9, \ln \left(1.7 \times 10^{14}\right)\right)$.

Having fixed the parameters to those fiducial values, we end up with a total of $\sim 1700000$ galaxies and $\sim 25000$ galaxy clusters. The redshift dependence of the comoving number density and the product of the real-space linear bias parameter $b(z)$ and the linear growth factor $g(z)$ (i.e. the linear bias parameter with respect to the $z=0$ matter power spectrum) is shown in the upper and lower panels of Fig. 2, respectively. In redshift space the product $b(z) g(z)$ gets boosted by a factor of 1.12 and reaches $\sim 2.1$ at redshift $z=0.1$ as compared to the corresponding realspace value of 1.85 .

The important ingredients of the Fisher matrix calculations are the weight factors $\left(w^{g}, w^{c}, w^{c-g}\right)$ and the logarithmic derivatives of the power spectra (see Eqs. (B.5)-(B.8)). The angleaveraged weight factors as functions of the comoving wavenumber $k$ and redshift $z$ for the galaxies, clusters, and cluster-galaxy cross pairs are shown as upper, middle, and lower panels of Fig. 3, respectively. There the discrete contour lines start from the value of 0.5 and increase with a constant step of 0.05 . As expected, we see that the number density of galaxies $(\sim 3 \times$ $\left.10^{-4} h^{3} \mathrm{Mpc}^{-3}\right)$ is sufficiently high to ensure a well-sampled density field (i.e. weight factors close to 1 ) for all of the wavenumbers and redshifts of interest. This is not the case for the galaxy clusters for which the high shot-noise contribution reduces the weights significantly, reaching the value of 0.5 on large scales

\footnotetext{
17 However, from the observational data, several authors have obtained values for $\alpha$ that are somewhat higher: Collister \& Lahav (2005) found $\alpha=1.05$ for the 2dFGRS red galaxies, Blake et al. (2008) found $\alpha$ for the LRGs to be evolving with redshift, from 1.57 to 1.80 .
}
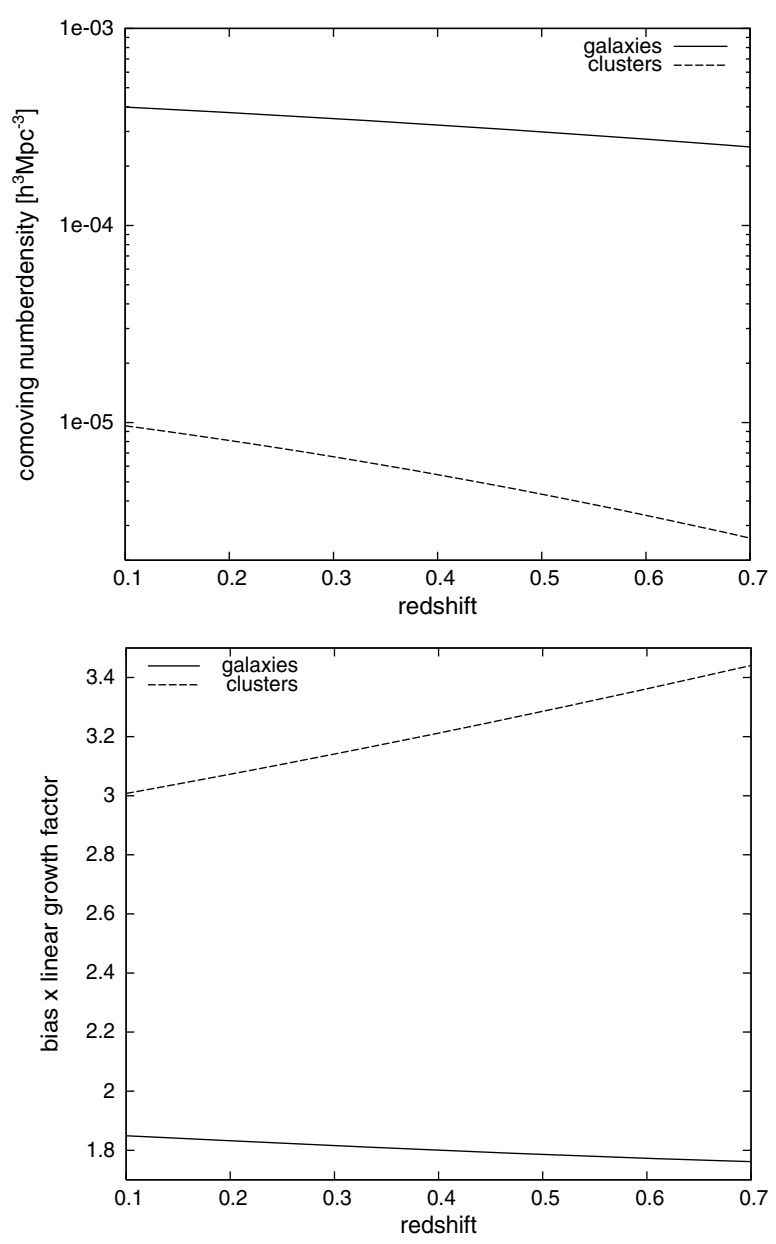

Fig. 2. Comoving number density (upper panel) and the product of the real-space bias parameter and linear growth factor $b(z) g(z)$ (lower panel) for galaxies (solid lines) and galaxy clusters (dashed lines) as a function of redshift.

only for the lowest redshifts. For the cluster-galaxy cross pairs, the situation is significantly better: here the weight factor is above the value of 0.5 for the most of the $k-z$ plane.

The logarithmic derivatives of the galaxy, cluster, and cross spectra are given in Fig. 4. We see that for most of the parameters the derivatives are very similar, which leads to almost identical degenerate parameter combinations. However, there are significant differences in logarithmic derivatives involving $\sigma_{8}{ }^{18}$ and HOD parameters $\alpha$ and $\ln m_{g}^{\text {low }}$, and thus for these one would expect tighter constraints once the extra information from the cross pairs is included. This is indeed the case as can be seen from Fig. 5 and Table 1. In Fig. 5 we have plotted the 2D error ellipses $(1-\sigma)$ and also 1D Gaussian distributions for the eight free parameters involved. There the largest ellipses correspond to the constraints from the cross pairs, middle ones to the galaxy-galaxy pairs, and the smallest to the combined $(g-g)+(c-g)$ case. In Table 1 we give the figures-of-merit (FOMs), defined as the inverse areas of the 1- $\sigma$ error ellipses, for several free parameter combinations. The diagonal entries (e.g. $\Omega_{m}-\Omega_{m}$ ) in this table give the inverse of the corresponding

18 Note the difference in sign of the logarithmic derivatives of the galaxy and cluster power spectra in this case. By increasing $\sigma_{8}$ the amplitude of the cluster power spectrum actually drops. This stems from the higher value of $\sigma_{8}$ leading to the rapid increase in the number of low-mass clusters that are less weakly biased. 

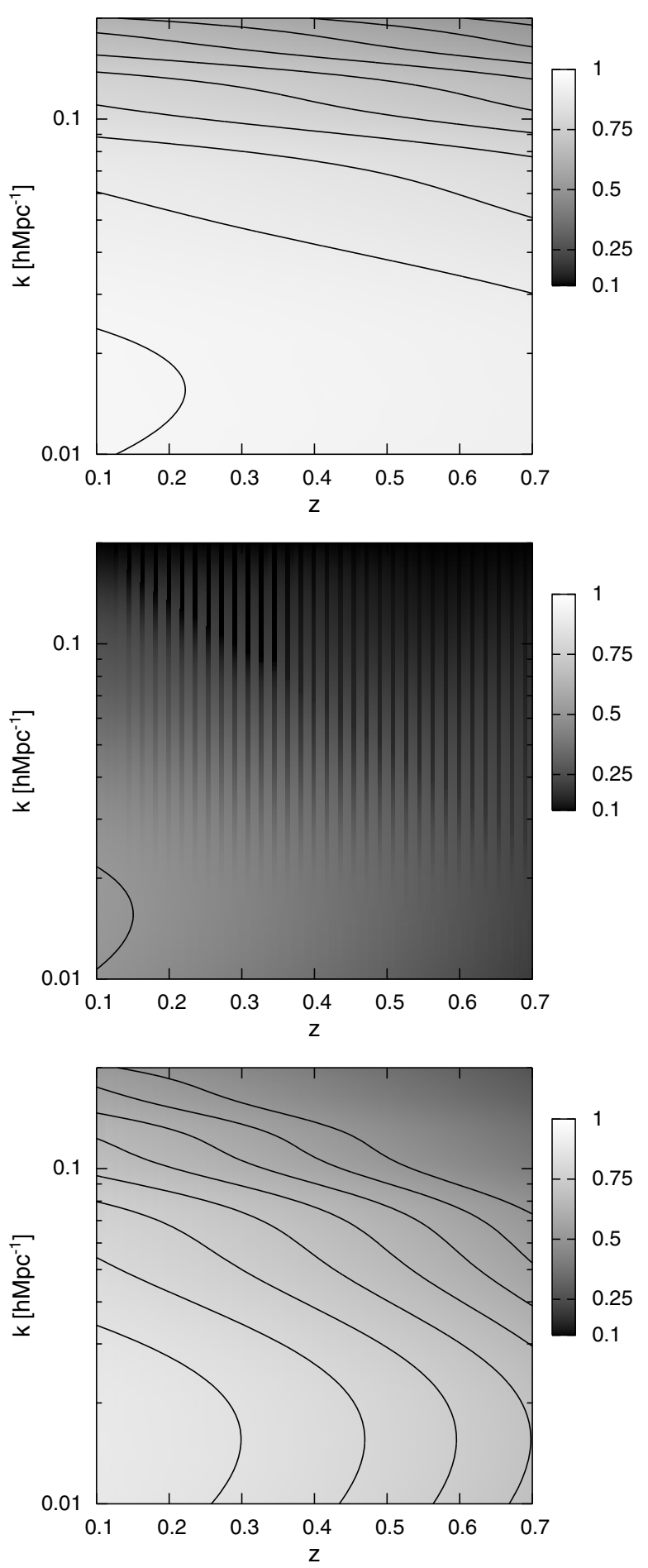

Fig. 3. The angle-averaged weight factors $w^{g}$ (upper panel), $w^{c}$ (middle panel), and $w^{c-g}$ (lower panel) as functions of the comoving wavenumber $k$ and redshift $z$. The discrete contour lines start from the value of 0.5 and increase with a constant step of 0.05 .

parameter's 1- $\sigma$ error interval, instead. In each cell the numbers from top to bottom correspond to $c-g, g-g,(g-g)+(c-g)$, and the ratio of $(g-g)+(c-g)$ and $g-g$ FOMs. In Table 1 we have decided not to show an additional gain once $c-c$ information is included, as this is relatively modest compared to the accuracy boost brought by including the cluster-galaxy cross pairs. As already mentioned, the biggest boost in accuracy, once cross pairs are included occurs for the parameter combinations involving $\sigma_{8}, \alpha$, and $\ln m_{g}^{\text {low }}$; e.g., the error ellipse for the $\sigma_{8}-w_{0}$ case gets reduced by a factor of $\sim 2.4$, etc. Since the $g-g$ spectrum does not depend on the ninth parameter $\ln m_{c}^{\text {low }}$, we decided not to include it in Fig. 5 and Table 1. We only note that, even without the assumed $10 \%$ prior for $m_{c}^{\text {low }}$, the information encoded in the $c-g$ pairs is able to self-calibrate $m_{c}^{\text {low }}$ to an accuracy of $\sim 4 \%$, and thus our results in Fig. 5 and Table 1 only change slightly once this extra prior is not applied.

In Fig. 6 we show in greater detail the improvement on the $m_{g}^{\text {low }}-\alpha$ plane by including of the information from the cross pairs. Although the constraints in the $c-g$ case are relatively weak, a different degeneracy direction from the $g-g$ case still helps in shrinking a $g-g$ error ellipse by a factor of $\sim 2$ (see Table 1). Also, as one of the main goals of these future redshift surveys is to put constraints on the properties of dark energy, Fig. 7 presents the performance on the $w_{0}-w_{a}$ plane in greater detail. The dark-energy FOMs we obtain are 26.9, 38.5, 28.8, and 40.4 for the $g-g,(g-g)+(c-g),(g-g)+(c-c)$, and $(g-g)+(c-c)+(c-g)$ cases, respectively ${ }^{19}$. Here we see that the extra information from the cross pairs leads to a relatively modest gain: error ellipse is compressed by a factor of $\sim 1.4$ as compared to the $g-g$ case taken separately. We also see that the additional gain induced by including of the cluster-cluster pairs is significantly smaller than that. This is also the case for the rest of the parameters, which is why we have not included $c-c$ case in Fig. 5 and Table 1.

In our Fisher-matrix calculations, we focused only on a wavenumber interval $k=0.01-0.2 \mathrm{~h} \mathrm{Mpc}^{-1}$. Due to formation of the nonlinear structures, the Gaussianity assumptions is not adequate for wavenumbers larger than $\sim 0.2 \mathrm{~h} \mathrm{Mpc}^{-1}$. On those small scales, the information is transferred out of the two-point function to the higher order moments, increasing the variance of the power spectrum above its Gaussian expectation; i.e., to calculate the variance one has to include a contribution from the trispectrum (see e.g. Scoccimarro et al. 1999). Moreover, there have been a few studies (e.g. Rimes \& Hamilton 2006; Neyrinck \& Szapudi 2007) that indicate that there is not much information left in the power spectrum on those scales. As we have chosen to divide our survey volume into relatively narrow redshift shells, it is clear that we are not able to probe the very large Fourier modes. However, the modes with wavenumbers above $0.01 h \mathrm{Mpc}^{-1}$ are still relatively well-sampled. We have checked that our results are rather insensitive to lowering the wavenumber boundary below its assumed value of $k=0.01 h \mathrm{Mpc}^{-1}$. For the $c-g$ case, this can be seen by looking at Fig. 8 where we have shown how Fisher information (for the diagonal matrix entries only) is distributed on the wavenumber-redshift plane, i.e. the total Fisher information is given by the integral over this plane. There the discrete contour lines correspond to the isocontours enclosing 50, 70, and 90\% of the total Fisher information. One can see that, for most of the parameters, the surface drops off rapidly towards large scales and thus $90 \%$ contour does not reach $k=0.01 h \mathrm{Mpc}^{-1}$ line. For the $g-g$ and $c-c$ case, the picture is qualitatively similar.

\section{Conclusions and discussion}

There are several wide-field galaxy and cluster surveys planned for the near future. In the simplest approach, one would analyze

\footnotetext{
${ }^{19}$ For the flat models with a scalar spectral index $n_{\mathrm{s}}=1$ (as was assumed so far) by adding extra information obtainable from the CMB measurements by the PLANCK satellite these FOMs increase to $81.9,125,86.1$, and 135 , respectively.
} 

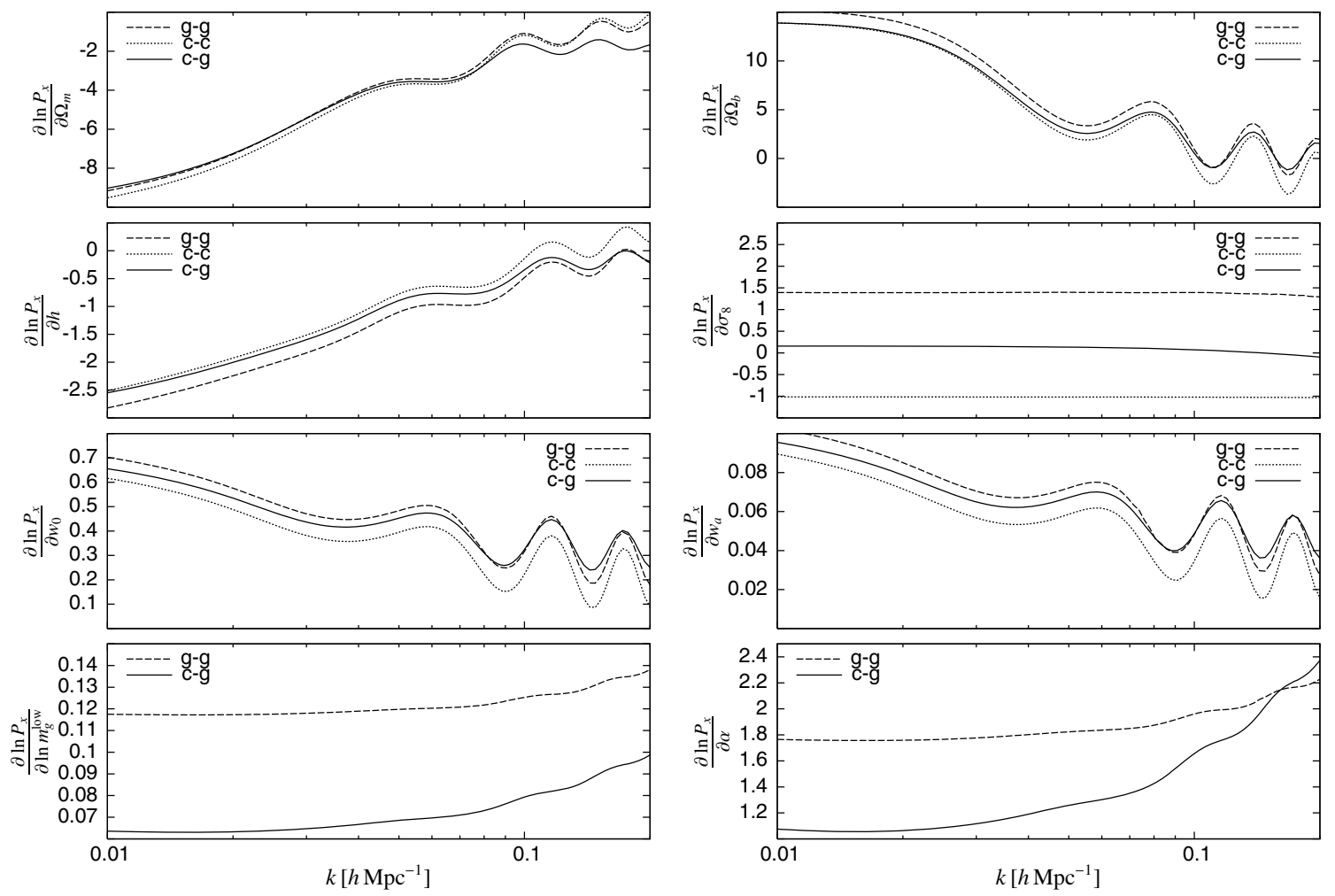

Fig. 4. Logarithmic derivatives of the galaxy (dashed lines), cluster (dotted lines), and cluster-galaxy power spectra.

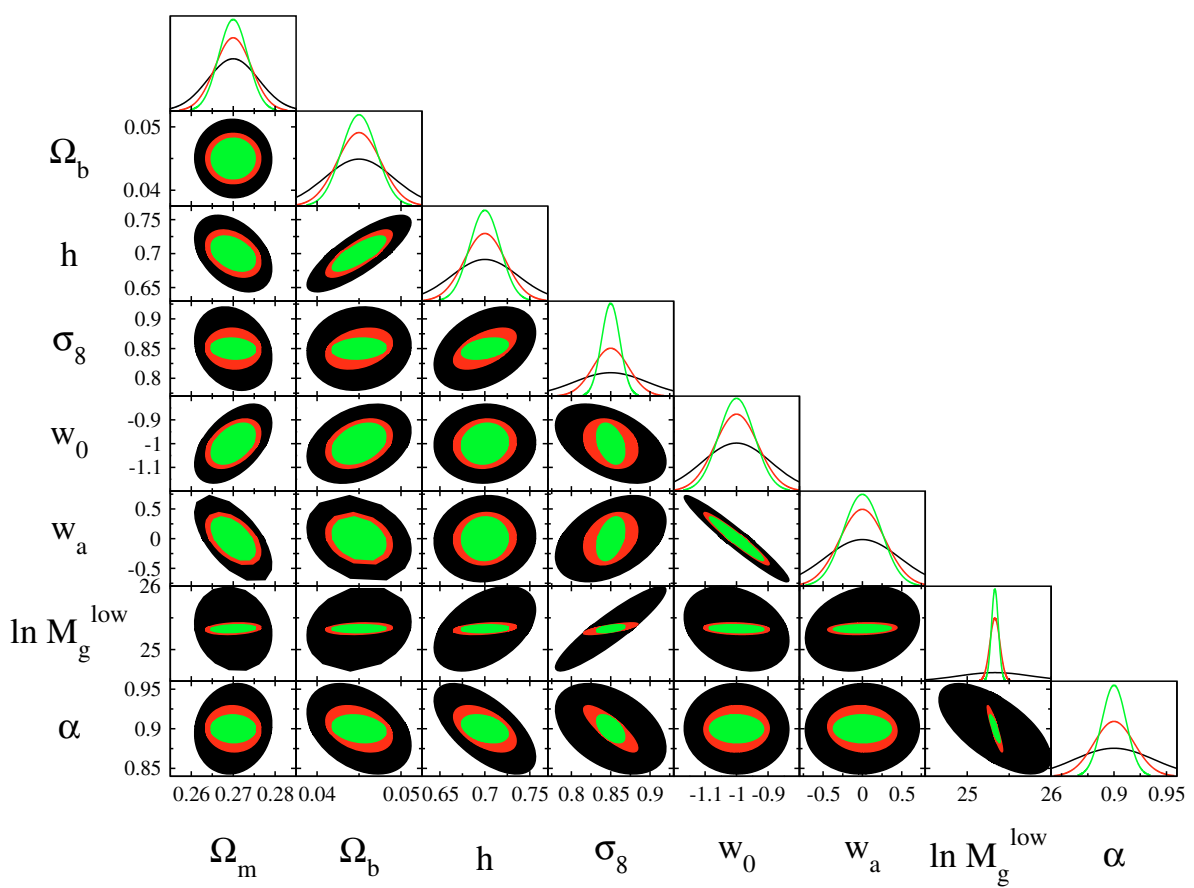

Fig. 5. 2D error ellipses (1- $\sigma$ ) and 1D Gaussian distributions for eight of the free model parameters. The largest ellipses correspond to the constraints from the cluster-galaxy cross pairs, middle ones to the galaxy-galaxy pairs, and the smallest to the combined $(g-g)+(c-g)$ case. these independently, thus neglecting the extra information provided by the cluster-galaxy cross pairs. In this paper we have focused on the possible synergy between these surveys by investigating the amount of information encoded in the cross pairs. Since in our study we focus on two-point clustering statistics, our approach complements the number count analysis, which in its usual form assumes validity of the Poissonian statistics ${ }^{20}$.

${ }^{20}$ In the case of the galaxy cluster samples, the complementarity of
the number count and clustering analysis has been investigated by
To model the cluster-galaxy cross spectrum we used the HM framework. We carried out a Fisher matrix analysis for a

Majumdar \& Mohr (2004). For example, these authors illustrate that combining number counts and $c-c$ power spectrum helps in reducing the error bar on $\sigma_{8}$ by a factor of two for an SPT-like survey. This is similar to the improvement on $\sigma_{8}$ we obtain by combining $c-g$ with $g-g$. Due to the sparseness of the cluster sample, the additional inclusion of the $c-c$ power leads to only mildly stronger constraints. To evaluate the absolute error on $\sigma_{8}$, one should combine our pair statistics with the cluster number counts, which is beyond the scope of the current work. 
Table 1. Figures-of-merit (FOMs), defined as the inverse areas of the $1-\sigma$ error ellipses.

\begin{tabular}{|c|c|c|c|c|c|c|c|c|}
\hline & $\Omega_{\mathrm{m}}$ & $\Omega_{\mathrm{b}}$ & $h$ & $\sigma_{8}$ & $w_{0}$ & $w_{a}$ & $\ln m_{g}^{\text {low }}$ & $\alpha$ \\
\hline \multirow{4}{*}{$\Omega_{m}$} & \begin{tabular}{|l|}
165 \\
\end{tabular} & 5610 & 673 & 506 & 244 & 60.4 & 52.5 & 612 \\
\hline & 232 & 12,300 & 1510 & 1420 & 523 & 129 & 546 & 1670 \\
\hline & 290 & 19,100 & 2570 & 3450 & 797 & 197 & 998 & 3470 \\
\hline & 1.25 & 1.55 & 1.70 & 2.43 & 1.53 & 1.53 & 1.83 & 2.07 \\
\hline \multirow{4}{*}{$\Omega_{\mathrm{b}}$} & & 245 & 1550 & 736 & 328 & 73.2 & 78.3 & 970 \\
\hline & - & 384 & 4020 & 2470 & 827 & 189 & 903 & 2950 \\
\hline & & 477 & 6580 & 5700 & 1250 & 282 & 1600 & 5850 \\
\hline & & 1.24 & 1.64 & 2.31 & 1.51 & 1.50 & 1.77 & 1.98 \\
\hline \multirow{4}{*}{$h$} & & & 26.9 & 87.2 & 34.0 & 7.81 & 9.49 & 127 \\
\hline & 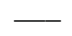 & - & 43.7 & 328 & 88.7 & 20.9 & 107 & 372 \\
\hline & & & 58.7 & 758 & 144 & 33.5 & 199 & 754 \\
\hline & & & 1.34 & 2.31 & 1.62 & 1.60 & 1.86 & 2.03 \\
\hline \multirow{4}{*}{$\sigma_{8}$} & & & & 21.6 & 30.8 & 6.88 & 16.9 & 87.5 \\
\hline & 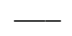 & - & - & 44.2 & 92.8 & 22.2 & 163 & 581 \\
\hline & & & & 85.6 & 226 & 53.2 & 344 & 1260 \\
\hline & & & & 1.94 & 2.44 & 2.39 & 2.12 & 2.17 \\
\hline \multirow{4}{*}{$w_{0}$} & & & & & 9.13 & 9.86 & 3.00 & 33.6 \\
\hline & 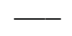 & - & - & - & 14.6 & 26.9 & 34.1 & 105 \\
\hline & & & & & 17.6 & 38.5 & 59.9 & 211 \\
\hline & & & & & 1.21 & 1.43 & 1.76 & 2.00 \\
\hline \multirow{4}{*}{$w_{a}$} & & & & & & 2.09 & 0.675 & 7.70 \\
\hline & & 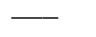 & - & - & - & 3.45 & 8.06 & 25.0 \\
\hline & & & & & & 4.12 & 13.9 & 49.3 \\
\hline & & & & & & 1.19 & 1.73 & 1.97 \\
\hline \multirow{4}{*}{$\ln m_{g}^{\text {low }}$} & & & & & & & 2.27 & 11.0 \\
\hline & - & - & - & $\longrightarrow$ & 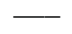 & - & 16.6 & 320 \\
\hline & & & & & & & 24.2 & 652 \\
\hline & & & & & & & 1.46 & 2.04 \\
\hline \multirow{3}{*}{$\alpha$} & & & & & & & & 26.6 \\
\hline & & & $\bar{\square}$ & - & & 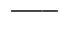 & 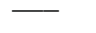 & 52.1 \\
\hline & & & & & & & & 1.66 \\
\hline
\end{tabular}

Note: the diagonal entries (e.g. $\Omega_{\mathrm{m}}-\Omega_{\mathrm{m}}$ ) give the inverse of the corresponding parameter's 1- $\sigma$ error interval, instead. In each cell the numbers from top to bottom correspond to $c-g, g-g,(g-g)+(c-g)$, and the ratio of $(g-g)+(c-g)$ and $g-g$ FOMs.

BOSS-like galaxy redshift survey targeting luminous red galaxies over $10000 \mathrm{deg}^{2}$ of sky and over the redshift range of $z=0.1-0.7$, and a hypothetical mass-limited cluster redshift survey (with a threshold mass $m_{c}^{\text {low }}=1.7 \times 10^{14} h^{-1} M_{\odot}$ ) over the same volume. For simplicity, we assumed spatially flat models with the usual $w_{0}-w_{a}$ parametrization for the dark-energy equation of state. For the HOD we used a simple description given by Eqs. (9)-(11). Since clustering does not depend on $m_{0}$, we are left with two HOD parameters: $\alpha$ and $m_{g}^{\text {low }}$. Adding to these $m_{c}^{\text {low }}$ and six cosmological parameters: $\Omega_{m}, \Omega_{b}, h, \sigma_{8}, w_{0}$, and $w_{a}$, we have nine free parameters in total.

On small scales the cluster-galaxy cross spectrum directly probes density profiles of the halos, instead of the profile convolved with itself, as is the case for the galaxy power spectrum. Because of this, we are sensitive to a different combination of the HOD parameters. This leads to stronger constraints on the HOD parameters $\alpha$ and $m_{g}^{\text {low }}$ as compared to the constraints obtainable from the galaxy data taken separately; e.g., the inclusion of the cross pairs leads to a factor of $\sim 2$ compression of the error ellipses on the $m_{g}^{\text {low }}-\alpha$ plane. Also, the extra information in the cross pairs leads to a factor of $\sim 2$ tighter constraint on $\sigma_{8}$. The best performance is obtained on $\sigma_{8}-\Omega_{\mathrm{m}}, \sigma_{8}-w_{0}$, and $\sigma_{8}-w_{a}$ planes where the error ellipses get reduced by a factor of $\sim 2.4$.

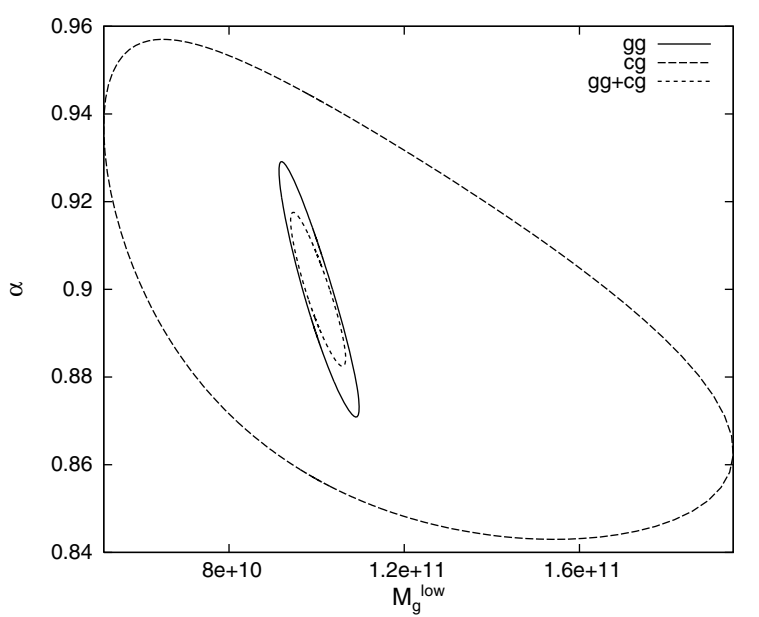

Fig. 6. $1-\sigma$ error contours on the $m_{g}^{\text {low }}-\alpha$ plane. Solid, dashed, and short-dashed lines correspond to the $g-g, c-g$, and $(g-g)+(c-g)$ cases, respectively.

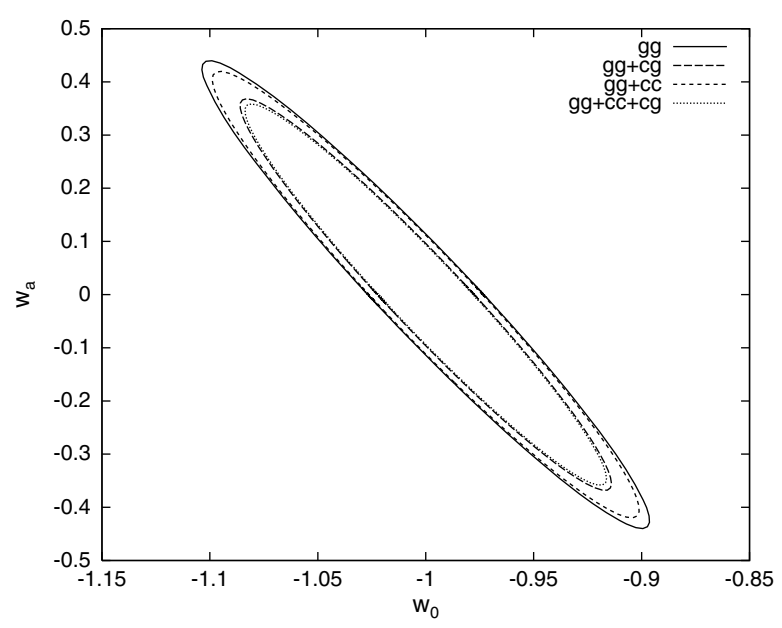

Fig. 7. 1- $\sigma$ error contours on the $w_{0}-w_{a}$ plane. Solid, dashed, shortdashed, and dotted lines correspond to the $g-g,(g-g)+(c-g)$, $(g-g)+(c-c)$, and $(g-g)+(c-c)+(c-g)$ cases, respectively.

For the dark-energy equation of state parameters $w_{0}$ and $w_{a}$, the improvement is somewhat weaker. Including the clustergalaxy cross pairs leads to a factor of $\sim 1.4$ increase in the darkenergy figure-of-merit as compared to the galaxy-galaxy case taken separately. For the rest of the results, see Fig. 5 and Table 1.

It is worth mentioning that the small-scale information in the one-halo term of the cluster-galaxy power spectrum is essentially equivalent to the information gained by directly fitting the galaxy distribution within the halos $\left(P_{c-g}^{1 h}\right.$ is just a Fourier transform of the weighted mean of the halo density profiles). From the relatively sparse data provided by the assumed redshift surveys, one cannot reliably perform profile-fitting on an object-byobject basis, and thus probably has to rely on stacking methods, which also lead to some sort of a weighted profile. Thus these two approaches should give essentially the same results.

In our analysis we assumed that the threshold cluster mass $m_{c}^{\text {low }}$ can be calibrated with an accuracy of $10 \%$. We find that, even without the assumed $10 \%$ prior for $m_{c}^{\text {low }}$, the information encoded in the $c-g$ pairs is able to self-calibrate $m_{c}^{\text {low }}$ to an accuracy of $\sim 4 \%$, and thus our results are insensitive to this additional prior. On the other hand, treating $m_{c}^{\text {low }}$ as a known quantity and fixing it to the fiducial value of $1.7 \times 10^{14} h^{-1} M_{\odot}$, 

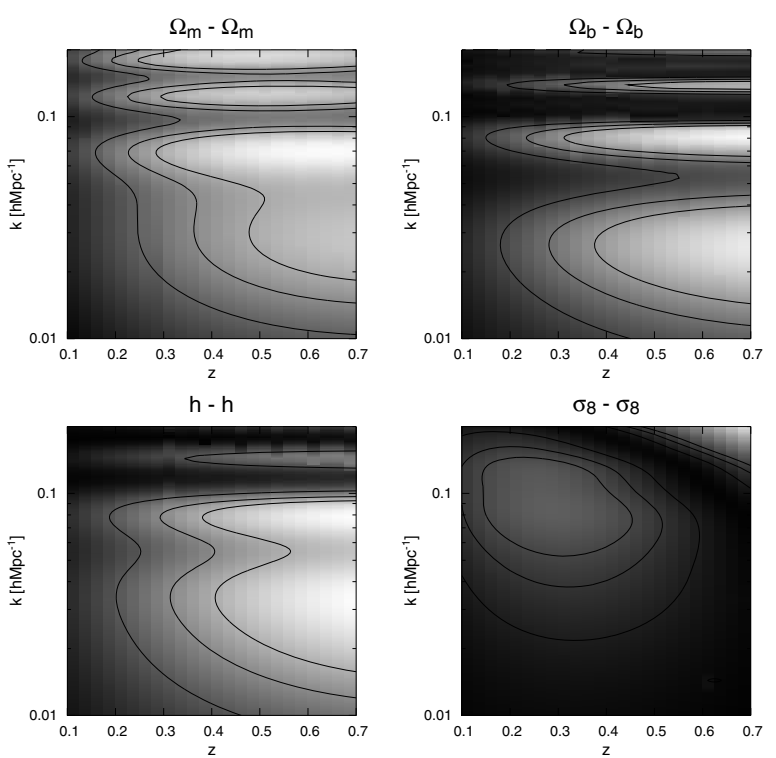

$w_{0}-w_{0}$
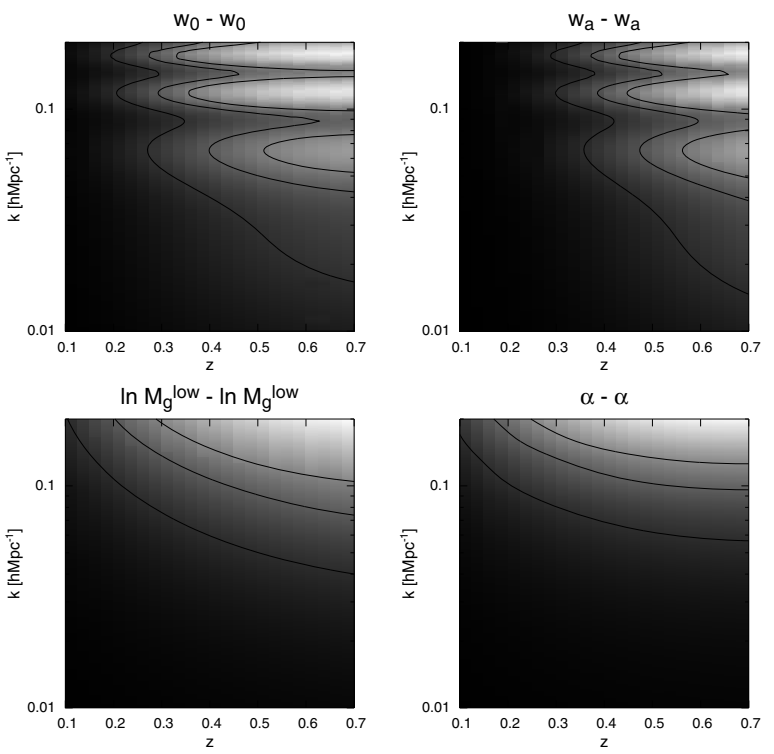

Fig. 8. Distribution of the $c-g$ Fisher information (for the diagonal matrix entries only) on the wavenumber-redshift plane, i.e. the total Fisher information is given by the integral over this plane. The discrete contour lines correspond to the isocontours enclosing 50, 70, and $90 \%$ of the total Fisher information.

we obtain $\sim 5$ times tighter constraint on $\sigma_{8}$. Similarly, all the error ellipses involving $\sigma_{8}$ as one of the parameters get reduced by factors of 6-10 in this case, the most remarkable being a factor of $\sim 10$ reduction of the error ellipse on the $\sigma_{8}-m_{g}^{\text {low }}$ plane. For the other parameters, the precise knowlwdge of $m_{c}^{\text {low }}$ leads to much milder improvements; e.g., $m_{g}^{\text {low }}-\alpha$ and $w_{0}-w_{a}$ error ellipses now decrease by factors of $\sim 3$ and $\sim 1.5$, respectively.

In our analysis we have simply added Fisher matrices corresponding to the galaxy-galaxy and cluster-galaxy pairs. This is a valid approach once the density field is not over sampled. If that is not the case, the extra pairs do not help in increasing the information; i.e., then we cannot simply add Fisher matrices. However, in our case the Fisher information comes mostly from the larger wavenumbers as there are simply more high$k$ modes available (Fig. 8 shows this for the $c-g$ case). As can be seen from Fig. 3, our hypothetical surveys are surely not over sampled for large wavenumbers, and thus simply adding the Fisher matrices seems to be a valid approximation.

As a final comment we point out that the formalism as presented in Sect. 2 can also be applied to the photometric redshift surveys such as DES, Pan-STARRS, DUNE, and LSST. In this case including cluster-galaxy pairs might be very beneficial since the photo- $z$ errors for the clusters are usually significantly smaller than for the typical galaxies.

\section{Appendix A: Power spectra in photometric redshift space}

As there will be several large imaging surveys performed in the future (e.g. DES, Pan-STARRS, DUNE, LSST), we present here a minimal extension of the formalism for the case when only photometric redshifts are available. For simplicity we assume that the conditional probability distribution for the spectroscopic redshift, given the photometric one $P\left(z_{\text {spec }} \mid z_{\text {photo }}\right)$, is a Gaussian with a mean value of $z_{\text {photo }}$ (i.e. we assume zero bias) and with dispersion $\delta z$. This leads to the extra damping factors for the density contrast, which have the same form as in Eq. (19) with the 1D spatial smoothing scale $\sigma$ given as $\sigma=\frac{c}{H_{0}} \delta z$. We denote the corresponding smoothing scales for clusters and galaxies as $\sigma_{c}^{\text {photo }}$ and $\sigma_{g}^{\text {photo }}$, respectively. As for any realistic case, photo- $z$ errors change with redshift, and so $\sigma_{c}^{\text {photo }}$ and $\sigma_{g}^{\text {photo }}$ are modeled as functions of $z$.

Thus for the dark matter, we have an effective smoothing scale $\sigma_{m}(m, z)$, for the galaxies $\sigma_{g}(m, z) \equiv$ $\sqrt{\left(\sigma_{m}(m, z)\right)^{2}+\left(\sigma_{g}^{\text {photo }}(z)\right)^{2}}(\mathrm{FOG}+$ photo- $z$ errors $)$, and for the clusters $\sigma_{c}(z) \equiv \sigma_{c}^{\text {photo }}(z)$ (photo-z errors only). We also define the quantity $\sigma_{c-g}(m, z) \equiv \sqrt{\left(\sigma_{c}(z)\right)^{2}+\left(\sigma_{g}(m, z)\right)^{2}}=$ $\sqrt{\left(\sigma_{m}(m, z)\right)^{2}+\left(\sigma_{c}^{\text {photo }}(z)\right)^{2}+\left(\sigma_{g}^{\text {photo }}(z)\right)^{2}}$. Having introduced these new smoothing scales, we can obtain results valid for the photometric redshift space, if we substitute $\sigma_{m}(m, z)$ with $\sigma_{g}(m, z)$ in Eqs. (24) and (33), $\sigma_{m}(m, z)$ with $\sigma_{c-g}(m, z)$ in Eq. (25), and include an additional factor of $\mathcal{F}\left[k \sigma_{c}(z) \mu\right]$ in Eq. (32).

\section{Appendix B: Covariances and Fisher matrices}

Under the assumption of Gaussianity the power spectrum covariance matrix can be expressed as (Feldman et al. 1994; Tegmark 1997)

${ }^{x} \mathbf{C}_{\mathrm{mn}} \simeq \frac{2 P_{x}^{2}\left(k_{n}\right)}{V_{n} V_{\mathrm{eff}}^{x}\left(k_{n}\right)} \delta_{\mathrm{mn}}$,

where $P_{x}\left(k_{n}\right)$ denotes the power spectrum of clusters/galaxies for the wavenumber bin centered at wavenumber $k_{n}, V_{n} \equiv$ $4 \pi k_{n}^{2} \Delta k_{n} /(2 \pi)^{3}\left(\Delta k_{n}\right.$ - width of the wavenumber bin) is the volume of the shell in Fourier space, and the effective volume $V_{\mathrm{eff}}^{x}(k)$ is given as

$V_{\mathrm{eff}}^{x}(k) \equiv \int\left[\frac{\bar{n}_{x}(\boldsymbol{r}) P_{x}(k)}{1+\bar{n}_{x}(\boldsymbol{r}) P_{x}(k)}\right]^{2} \mathrm{~d} \boldsymbol{r}=\left[\frac{\bar{n}_{x} P_{x}(k)}{1+\bar{n}_{x} P_{x}(k)}\right]^{2} V$,

where $V$ is the total volume of the survey. Here the second equality for $V_{\mathrm{eff}}(k)$ is valid only if the selection function of the cluster/galaxy sample, $\bar{n}_{x}(\boldsymbol{r})$, is independent of position.

Following the lines of thought in Appendix B of Feldman et al. (1994), which led to the result given in Eq. (B.1) we find 
that the similar result for the cluster-galaxy cross spectrum can be expressed as

${ }^{c-g} \mathbf{C}_{m n} \simeq\left[\frac{P_{c-g}^{2}\left(k_{n}\right)}{V_{n} V}+\frac{P_{c-c}\left(k_{n}\right) P_{g-g}\left(k_{n}\right)}{V_{n} V_{\mathrm{eff}}^{c-g}\left(k_{n}\right)}\right] \delta_{m n}$,

where the effective volume $V_{\mathrm{eff}}^{c-g}(k)$,

$$
\begin{aligned}
V_{\mathrm{eff}}^{c-g}(k) & \equiv \int\left[\frac{\bar{n}_{c}(\boldsymbol{r}) P_{c-c}(k)}{1+\bar{n}_{c}(\boldsymbol{r}) P_{c-c}(k)}\right]\left[\frac{\bar{n}_{g}(\boldsymbol{r}) P_{g-g}(k)}{1+\bar{n}_{g}(\boldsymbol{r}) P_{g-g}(k)}\right] \mathrm{d} \boldsymbol{r} \\
& =\left[\frac{\bar{n}_{c} P_{c-c}(k)}{1+\bar{n}_{c} P_{c-c}(k)}\right]\left[\frac{\bar{n}_{g} P_{g-g}(k)}{1+\bar{n}_{g} P_{g-g}(k)}\right] V .
\end{aligned}
$$

The first term in Eq. (B.3) is the analog of Eq. (B.1) with the difference of the missing factor of two; also here, instead of the effective volume, we have a survey volume $V$, which is due to not having a shot-noise term from self-pairs. To find the corresponding Fisher matrices we follow the derivation in Tegmark (1997). In our calculations we divide the survey volume into relatively narrow redshift bins. This allows us to approximate the spatial number densities of objects as constant quantities within the bins; i.e., we can use the second equalities of Eqs. (B.2) and (B.4).

The Fisher matrices for the anisotropic cluster/galaxy spectra can be given as

$$
\begin{aligned}
{ }^{x} \mathbf{F}_{i j}\left(z_{n}\right) \simeq & \frac{V\left(z_{n}\right)}{8 \pi^{2}} \cdot \int_{-1}^{1} \mathrm{~d} \mu \int_{k_{\min }}^{k_{\max }} \mathrm{d} k k^{2} w^{x}\left(k, \mu \mid z_{n}\right) \\
& \frac{\partial \ln P_{x}\left(k, \mu \mid z_{n}\right)}{\partial \Theta_{i}} \cdot \frac{\partial \ln P_{x}\left(k, \mu \mid z_{n}\right)}{\partial \Theta_{j}},
\end{aligned}
$$

$w^{x}\left(k, \mu \mid z_{n}\right) \equiv\left[\frac{\bar{n}_{x}\left(z_{n}\right) P_{x}\left(k, \mu \mid z_{n}\right)}{1+\bar{n}_{x}\left(z_{n}\right) P_{x}\left(k, \mu \mid z_{n}\right)}\right]^{2}=\frac{V_{\mathrm{eff}}^{x}\left(k, \mu \mid z_{n}\right)}{V\left(z_{n}\right)}$.

Here $z_{n}$ denotes the central redshift and $V\left(z_{n}\right)$ the comoving volume corresponding to the $n$th redshift bin.

The corresponding results for the cluster-galaxy cross spectrum are given as

$$
\begin{aligned}
{ }^{c-g} \mathbf{F}_{i j}\left(z_{n}\right) \simeq & \frac{V\left(z_{n}\right)}{8 \pi^{2}} \cdot \int_{-1}^{1} \mathrm{~d} \mu \int_{k_{\min }}^{k_{\max }} \mathrm{d} k k^{2} w^{c-g}\left(k, \mu \mid z_{n}\right) \\
& \cdot \frac{\partial \ln P_{c-g}\left(k, \mu \mid z_{n}\right)}{\partial \Theta_{i}} \cdot \frac{\partial \ln P_{c-g}\left(k, \mu \mid z_{n}\right)}{\partial \Theta_{j}},
\end{aligned}
$$

$w^{c-g}\left(k, \mu \mid z_{n}\right) \equiv \frac{2}{1+\frac{\left[1+\bar{n}_{c}\left(z_{n}\right) P_{c-c}\left(k, \mu \mid z_{n}\right)\right]\left[1+\bar{n}_{g}\left(z_{n}\right) P_{g-g}\left(k, \mu \mid z_{n}\right)\right]}{\bar{n}_{c}\left(z_{n}\right) \bar{n}_{g}\left(z_{n}\right) P_{c-g}^{2}\left(k, \mu \mid z_{n}\right)}}$.

The total Fisher matrices are then the sums over the ones corresponding to different redshift bins:

$$
{ }^{x} \mathbf{F}_{i j}=\sum_{n}{ }^{x} \mathbf{F}_{i j}\left(z_{n}\right)
$$

In the above relations $\Theta_{i}$ represents the $i$ th component of the parameter vector $\boldsymbol{\Theta}$, which consists of cosmological plus HM parameters in our case. According to the Cramér-Rao inequality, there does not exist an unbiased method that can measure the $i$ th parameter $\Theta_{i}$ with error bars less than $1 / \sqrt{\mathbf{F}_{i i}}$. If the other parameters are not known but estimated from the data as well, the minimum achievable error is given by $\sqrt{\mathbf{F}_{i i}^{-1}}$ instead.

\section{References}

Alcock, C., \& Paczynski, B. 1979, Nature, 281, 358

Bahcall, N. A., Ostriker, J. P., Perlmutter, S., \& Steinhardt, P. J. 1999, Science, 284,1481

Blake, C., \& Glazebrook, K. 2003, ApJ, 594, 665

Blake, C., Collister, A., Bridle, S., \& Lahav, O. 2007, MNRAS, 374, 1527

Blake, C., Collister, A., \& Lahav, O. 2008, MNRAS, 385, 1257

Bullock, J. S., Kolatt, T. S., Sigad, Y., et al. 2001, MNRAS, 321, 559

Chevallier, M., \& Polarski, D. 2001, Int. J. Mod. Phys. D, 10, 213

Cole, S., Percival, W. J., Peacock, J. A., et al. 2005, MNRAS, 362, 505

Collister, A. A., \& Lahav, O. 2005, MNRAS, 361, 415

Cooray, A. 2004, MNRAS, 348, 250

Cooray, A., \& Sheth, R. 2002, Phys. Rep., 372, 1

Dunkley, J., Komatsu, E., Nolta, M. R., et al. 2008, ArXiv e-prints, 803

Eisenstein, D. J., \& Hu, W. 1998, ApJ, 496, 605

Eisenstein, D. J., Zehavi, I., Hogg, D. W., et al. 2005, ApJ, 633, 560

Feldman, H. A., Kaiser, N., \& Peacock, J. A. 1994, ApJ, 426, 23

Hinshaw, G., Weiland, J. L., Hill, R. S., et al. 2008, ArXiv e-prints, 803

Hu, W., \& Haiman, Z. 2003, Phys. Rev. D, 68, 063004

Hütsi, G. 2006a, A\&A, 449, 891

Hütsi, G. 2006b, A\&A, 459, 375

Kaiser, N. 1987, MNRAS, 227, 1

Lilje, P. B., \& Efstathiou, G. 1988, MNRAS, 231, 635

Linder, E. V. 2003, Phys. Rev. D, 68, 083504

Majumdar, S., \& Mohr, J. J. 2004, ApJ, 613, 41

Navarro, J. F., Frenk, C. S., \& White, S. D. M. 1997, ApJ, 490, 493

Neyrinck, M. C., \& Szapudi, I. 2007, MNRAS, 375, L51

Padmanabhan, N., Schlegel, D. J., Seljak, U., et al. 2007, MNRAS, 378, 852

Peebles, P. J. E. 1974, ApJS, 28, 37

Percival, W. J., Baugh, C. M., Bland-Hawthorn, J., et al. 2001, MNRAS, 327, 1297

Percival, W. J., Nichol, R. C., Eisenstein, D. J., et al. 2007, ApJ, 657, 645

Rimes, C. D., \& Hamilton, A. J. S. 2006, MNRAS, 371, 1205

Scoccimarro, R., Zaldarriaga, M., \& Hui, L. 1999, ApJ, 527, 1

Seldner, M., \& Peebles, P. J. E. 1977a, ApJ, 214, L1

Seldner, M., \& Peebles, P. J. E. 1977b, ApJ, 215, 703

Seljak, U. 2000, MNRAS, 318, 203

Seljak, U. 2001, MNRAS, 325, 1359

Seo, H., \& Eisenstein, D. J. 2003, ApJ, 598, 720

Sheth, R. K., \& Tormen, G. 1999, MNRAS, 308, 119

Sheth, R. K., Mo, H. J., \& Tormen, G. 2001, MNRAS, 323, 1

Sunyaev, R. A., \& Zeldovich, Y. B. 1972, Comm. Astrophys. Space Phys., 4, 173

Sunyaev, R. A., \& Zeldovich, I. B. 1980, ARA\&A, 18, 537

Tegmark, M. 1997, Phys. Rev. Lett., 79, 3806

Tegmark, M., Blanton, M. R., Strauss, M. A., et al. 2004, ApJ, 606, 702

Tegmark, M., Eisenstein, D. J., Strauss, M. A., et al. 2006, Phys. Rev. D, 74, 123507

White, M. 2001, MNRAS, 321, 1 